\title{
Convex Geometry Perspective on the (Standard Model) Effective Field Theory Space
}

\author{
Cen Zhang* \\ Institute for High Energy Physics, and School of Physical Sciences, University of Chinese Academy of Sciences, Beijing 100049, China \\ and Center for High Energy Physics, Peking University, Beijing 100871, China \\ Shuang-Yong Zhou \\ Interdisciplinary Center for Theoretical Study, University of Science and Technology of China, Hefei, Anhui 230026, China \\ and Peng Huanwu Center for Fundamental Theory, Hefei, Anhui 230026, China
}

(Received 16 May 2020; accepted 8 October 2020; published 12 November 2020)

\begin{abstract}
We present a convex geometry perspective to the effective field theory (EFT) parameter space. We show that the second $s$ derivatives of the forward EFT amplitudes form a convex cone, whose extremal rays are closely connected with states in the UV theory. For tree-level UV completions, these rays are simply theories with all UV particles living in at most one irreducible representation of the symmetries of the theory. In addition, all the extremal rays are determined by the symmetries and can be systematically identified via group theoretical considerations. The implications are twofold. First, geometric information encoded in the EFT space can help reconstruct the UV completion. In particular, we will show that the dim8 operators are important in reverse engineering the UV physics from the standard model EFT and, thus, deserve more theoretical and experimental investigations. Second, theoretical bounds on the Wilson coefficients can be obtained by identifying the boundaries of the cone and are, in general, stronger than the current positivity bounds. We show explicit examples of these new bounds and demonstrate that they originate from the scattering amplitudes corresponding to entangled states.
\end{abstract}

DOI: 10.1103/PhysRevLett.125.201601

Introduction.-Effective field theory (EFT) is an important framework to systematically parameterize new highscale phenomena. Absent any clear signature of new particles from the LHC data, the standard model EFT (SMEFT) [1-3] has become a standard tool for studying indirect signs of new physics. If EFT operators are detected and the corresponding Wilson coefficients measured, the next step is to pin down the underlying UV theory. While determining the Wilson coefficients from a given UV theory is a systematized procedure [4-14], this inverse problem can be highly nontrivial, as one set of coefficients can be UV completed in many ways.

A geometric perspective provides hints to this problem. Consider the subspace of the EFT parameters [15], spanned by the operators that contribute to the second $s$ derivatives of the forward 2-to-2 scattering amplitude. The Wilson coefficients are subject to positivity bounds [16] (see [17-25] for earlier works and recent generalizations; also see the applications in SMEFT [26-30] and other areas [31-50]) for the EFT to have a UV completion that satisfies the axiomatic principles of quantum field theory.

Published by the American Physical Society under the terms of the Creative Commons Attribution 4.0 International license. Further distribution of this work must maintain attribution to the author(s) and the published article's title, journal citation, and DOI. Funded by SCOAP .
These bounds on dim- 8 operators are a set of linear homogeneous inequalities of the coefficients. The solutions form a convex cone whose vertex is the origin of the (linear) space spanned by the coefficients. In this Letter, we establish a connection between the geometry of the $s^{2}$ subspace of EFT and the UV physics behind. On the geometry side, the physical space is a convex cone that can be generated as positively weighted sums of its edges, i.e., its extremal rays (ERs). On the physics side, an ER corresponds to an irreducible representation (irrep) under the symmetries of the theory and can be obtained only by integrating out heavy states from this single irrep. This geometric view helps determine the UV physics from measurements. By using the convex nature of the subspace, one can often draw striking conclusions about the existence of states including their quantum numbers and couplings.

In SMEFT, dim-8 operators [29,51-53] linearly furnish this subspace. While dim- 6 coefficients are expected to be more accurately measured, they alone are insufficient to determine UV models: There is an infinite number of models, or combinations of UV states, that leave no net dim- 6 effect. A UV model can be determined only modulo the addition of these combinations. This is in contrast to dim-8, as positivity bounds imply that all UV completions must have dim- 8 effects $[16,26]$. The dim- 8 operators have attracted increasing attention as the LHC has accumulated more and more data. Various motivations for going beyond 
dim-6 have been discussed, e.g., in Refs. [28,30,41,54-59]. A number of dim- 8 coefficients can be tested at the TeV level at the LHC [56,59-62], while better sensitivities are expected at future colliders [58,63]. Furthermore, observables and opportunities that allow disentangling dim- 8 effects from the dim- 6 ones exist and are being studied $[52,59,64]$. We will show that the geometric connection to the UV physics gives another important motivation to study dim-8 operators: Their coefficients contain vital information for a bottom-up reconstruction of UV physics.

To formulate this mapping between ERs and UV states, an accurate description of the EFT cone is mandatory. The current positivity bound approach is not sufficient. Instead, we will take a different approach that follows the extremal representation [65] of convex cones. Before proceeding, it is instructive to introduce some basic concepts and facts in convex geometry.

A convex cone is a subset of a linear space that is closed under additions and positive scalar multiplications. An ER of a convex cone $\mathcal{C}_{0}$ is an element $x \in \mathcal{C}_{0}$ that cannot be split into two other elements in a nontrivial way; i.e., if we write $x=y_{1}+y_{2}$ with $y_{1}, y_{2} \in \mathcal{C}_{0}$, we must have $x=\lambda y_{1}$ or $x=\lambda y_{2}, \lambda$ being real constant. For example, the ERs of a polyhedral cone are its edges. The dual cone $\mathcal{C}_{0}^{*}$ of $\mathcal{C}_{0}$ is the set $\mathcal{C}_{0}^{*} \equiv\left\{y \mid x \cdot y \geq 0, \quad \forall x \in \mathcal{C}_{0}\right\}$, where $\cdot$ means the inner product of two vectors. We have $\left(\mathcal{C}_{0}^{*}\right)^{*}=\mathcal{C}_{0}$, and $\mathcal{C}_{1} \subset \mathcal{C}_{2}$ implies $\mathcal{C}_{1}^{*} \supset \mathcal{C}_{2}^{*}$. The full set of positive linear combinations of elements in some set $\mathcal{X}$ form a convex cone, denoted by cone $(\mathcal{X})$. Its ERs are a subset of $\mathcal{X}$.

EFT amplitudes as convex cones. - Consider the forward scattering amplitude $M_{i j \rightarrow k l}(s, t=0)$, where $s, t$ are the standard Mandelstam variables and $1 \leq i, j, k, l \leq n$ represent the low-energy modes. Using analyticity of $M_{i j \rightarrow k l}(s)$ and the generalized optical theorem, we have the following dispersion relation:

$$
\begin{aligned}
M^{i j k l} & =\int_{(\epsilon \Lambda)^{2}}^{\infty} \frac{d \mu \operatorname{Disc} M_{i j \rightarrow k l}(\mu)}{2 i \pi\left(\mu-\frac{M^{2}}{2}\right)^{3}}+(j \leftrightarrow l)+\text { c.c. } \\
& =\int_{(\epsilon \Lambda)^{2}}^{\infty} \sum_{X}^{\prime} \sum_{K=R, I} \frac{d \mu m_{K}^{i j} m_{K X}^{k l}}{\pi\left(\mu-\frac{M^{2}}{2}\right)^{3}}+(j \leftrightarrow l) .
\end{aligned}
$$

Here we have focused on particles with equal masses, $M^{2}$ being the total mass squared, and the lhs is the second-order $s$ derivative of $M_{i j \rightarrow k l}(s)$, with the low-energy discontinuity subtracted up to $\epsilon \Lambda$, a scale smaller than the EFT cutoff (see Supplemental Material [66] for more details and cases with different masses). ( $j \leftrightarrow l$ ) means all the previous terms with the swap $j \leftrightarrow l$. $\sum_{X}^{\prime}$ denotes the sum over possible $X$ states along with their phase spaces, and we have written the $i j \rightarrow X$ amplitude $M_{i j \rightarrow X} \equiv m_{R X}^{i j}+i m_{I X}^{i j}$.

The elastic version of this relation $(i=k, j=l)$ has been widely used to derive positivity bounds (because $m_{K X}^{i j} m_{K X}^{i j} \geq 0$; see, e.g., [16]). One may also mix different polarizations [26,27,31,35] and different particles (e.g., [26$30,50,67])$ to get more bounds by using $M^{i j k l} u^{i} v^{j} u^{k} v^{l} \geq 0$ [because $u^{i} v^{j} u^{k} v^{l} m_{K X}^{i j} m_{K X}^{k l}=\left(u^{i} m_{K_{X}}^{i j} v^{j}\right)^{2} \geq 0$ ], where $u^{i}$ and $v^{j}$ enumerate the particles and polarizations [68]. This can be viewed as the positivity bound from superposed states $u^{i}|i\rangle$ and $v^{j}|j\rangle$. In any case, the $M^{i j k l}$ on the lhs is a lowenergy quantity and can be expressed in terms of the Wilson coefficients, either at tree level or loop level, and we will use it as a proxy of the EFT space. At the tree level, $M^{i j k l}$ can be linearly mapped to the dim-8 coefficient space [26-30], so in the SMEFT discussions we will not distinguish the two. Note that, since our discussion will be based on $M^{i j k l}$, which is a physical object, field redefinitions and renormalization group (RG) running will not change our conclusions. The approach is generically applicable to any EFT, including the Higgs EFT, in case the latter is needed to describe $M^{i j k l}$.

Our goal is a more accurate characterization of the set $\mathcal{C}$ of all possible $M^{i j k l}$. The main observation is that Eq. (2) defines $\mathcal{C}$ as a convex cone. To see this, note that Eq. (2) represents a positively weighted sum of $m_{K X}^{i j} m_{K X}^{k l}+$ $(j \leftrightarrow l)$, with integration regarded as a limit of summation. For a model-independent EFT, $m_{K X}^{i j}$ are arbitrary $n \times n$ real matrices. Thus, the set $\mathcal{C}$ can be viewed as a convex cone

$$
\mathcal{C}=\operatorname{cone}\left(\left\{M \mid M^{i j k l}=m^{i(j} m^{|k| l)}, m \in \mathbb{R}^{n^{2}}\right\}\right) ;
$$

i.e., $\mathcal{C}$ is positively generated from all tensors of the form $m^{i(j} m^{|k| l)}$, where $i(j|k| l)$ means $j, l$ indices are symmetrized. Furthermore, $\mathcal{C}$ is a salient cone; i.e., if $c \in \mathcal{C}, c \neq 0$, then $-c \notin \mathcal{C}$. This is because any nonzero element of $\mathcal{C}$, after contracting with $\delta^{i k} \delta^{j l}$, is positive as $m^{i j} m^{i j}>0$. According to the Krein-Milman theorem [65], $\mathcal{C}$ is then determined by the convex hull of its ERs, which leads to the extremal representation of $\mathcal{C}$.

Before moving forward, we comment on the incompleteness of the elastic positivity bounds from superposed states. As they are derived using $M^{i j k l} u^{i} v^{j} u^{k} v^{l} \geq 0$, these bounds describe the dual cone of $\mathcal{Q} \equiv \operatorname{cone}\left(\left\{u^{i} v^{j} u^{k} v^{l}\right\}\right)$. If $\mathcal{Q}=\mathcal{C}^{*}$, then $\mathcal{Q}^{*}$ is an accurate description of $\mathcal{C}$. However, we will show explicit examples where $\mathcal{C}^{*}$ contains more elements than $\mathcal{Q}$, which implies that elastic bounds are not tight. In this respect, finding the extremal representation of $\mathcal{C}$ is a better approach.

ERs and UV states.-The ERs can be found by using symmetries. The forward scattering is invariant under an SO (2) rotation around the forward direction. Taking the SM as an example, we can rewrite the rhs of Eq. (2), choosing the intermediate states $X$ as irreps (denoted by $\mathbf{r}$ ) under the $\mathrm{SO}(2)$ rotation and the $\mathrm{SU}(3)_{C} \times \mathrm{SU}(2)_{L} \times \mathrm{U}(1)_{Y}$ symmetries. The Wigner-Eckart theorem dictates that $M\left(i j \rightarrow X^{\alpha}\right)$ can be written as $\langle X|\mathcal{M}| \mathbf{r}\rangle C_{i, j}^{r, \alpha}$, where $\alpha$ labels the states of $\mathbf{r}$ and $C_{i, j}^{r, \alpha}$ is the Clebsch-Gordan coefficients for the direct sum decomposition of $\mathbf{r}_{i} \otimes \mathbf{r}_{j}$, with $\mathbf{r}_{i}\left(\mathbf{r}_{j}\right)$ the irrep of $i(j)$. 
The dynamics is contained in $\langle X|\mathcal{M}| \mathbf{r}\rangle$, independent of $\alpha$. Equation (2) becomes

$$
M^{i j k l}=\int_{(\epsilon \Lambda)^{2}}^{\infty} d \mu \sum_{X \text { in } \mathbf{r}} \frac{|\langle X|\mathcal{M}| \mathbf{r}\rangle|^{2}}{\pi\left(\mu-\frac{1}{2} M^{2}\right)^{3}} P_{r}^{i(j|k| l)}
$$

where $P_{r}^{i j k l} \equiv \sum_{\alpha} C_{i, j}^{r, \alpha}\left(C_{k, l}^{r, \alpha}\right)^{*}$ are the projective operators of the $\mathbf{r}$ representation. Similar to Eq. (3), we identify the cone $\mathcal{C}$ as cone $\left(\left\{P_{r}^{i(j|k| l)}\right\}\right)$, and its ERs are a subset of $\left\{P_{r}^{i(j|k| l)}\right\}$. These $j, l$-symmetrized projectors are not necessarily extremal, so we call them potential ERs (PERs); taking their convex hull identifies the true ERs among them. $\mathcal{C}$ is determined by the ERs.

The ERs are closely related to UV completions. For a physics amplitude $M^{i j k l}$ to be extremal, on the rhs of Eq. (4), only one irrep can exist; otherwise, $M^{i j k l}$ can be written as a sum of two different elements of $\mathcal{C}$, which is nonextremal. This contains important information about the UV dynamics. For tree-level UV completions, an ER implies that its entire $M^{i j k l}$ can be generated from the exchange of a single (multiplet) particle; i.e., the theory is a "one-particle extension" of the SM. It may be generated by several particles, but they must all live in the same irrep and have the same interaction. For loop-level UV completions, similarly, all multiparticle intermediate states (which may include SM particles if RG effects are not negligible) have to live in a single irrep. For nonperturbative UV completions, subtleties may arise, but a similar inference might exist, if $M^{i j k l}$ can be interpreted as coming from the exchange of UV states. We, however, leave the nonperturbative cases for a future discussion. More generally, any point in $\mathcal{C}$ is a positive sum of the ERs, and this coincides with the decomposition of the intermediate UV states into irreps. Therefore, geometric information in $\mathcal{C}$ helps UV reconstruction.

This approach can be applied to subsets of particles closed under all symmetries. The PERs continue to be projective in this subspace, so results derived (such as bounds) are valid, in general. In the following, we will illustrate our approach with three subsets of SM fields: scalars, vectors, and fermions. For SM particles living in one multiplet, the number of PERs is finite, and $\mathcal{C}$ is polyhedral following a theorem by Minkowski and Weyl [69,70], which are easy to obtain. If more particles are involved, one may resort to more efficient numerical algorithms, such as the reverse search algorithm [71,72] for obtaining bounds, or simply classical linear programing methods, for testing the inclusion of given points [68].

The Higgs triangular cone.-The SM Higgs boson lives in the 2 of $\mathrm{SU}(2)_{L}$ and carries hypercharge 1/2. To find the PERs, we work with real scalars, define

$$
H=\left(\begin{array}{c}
\phi_{2}+i \phi_{1} \\
\phi_{4}-i \phi_{3}
\end{array}\right), \quad C=\left(\begin{array}{cc}
0 & \mathbf{1}_{2 \times 2} \\
-\mathbf{1}_{2 \times 2} & 0
\end{array}\right),
$$

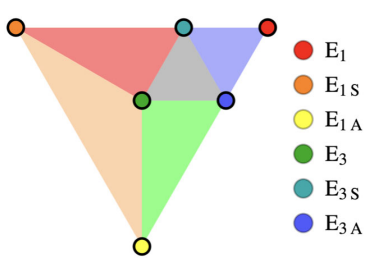

FIG. 1. A cross section of the Higgs triangular cone with the PERs, taken to be perpendicular to the direction $E_{1}+E_{1 S}+E_{1 A}$.

and use the $\gamma$ matrices defined in Ref. [73]. The projectors of the irreps from $\mathbf{2} \otimes \mathbf{2}$ define the following PERs:

$E_{1}^{i j k l}=\frac{1}{2}\left[C^{i(j} C^{|k| l)}+\left(C \gamma_{4}\right)^{i(j}\left(C \gamma_{4}\right)^{|k| l)}\right]$,

$E_{1 S}^{i j k l}=\mathbf{1}_{4 \times 4}^{i(j} \mathbf{1}_{4 \times 4}^{|k| l)}, E_{1 A}=\gamma_{4}^{i(j} \gamma_{4}^{|k| l)}$,

$E_{3}^{i j k l}=\frac{1}{2}\left[\left(C \gamma_{I}\right)^{i(j}\left(C \gamma_{I}\right)^{|k| l)}+\left(C \gamma_{4} \gamma_{I}\right)^{i(j}\left(C \gamma_{4} \gamma_{I}\right)^{|k| l)}\right]$

$E_{3 S}^{i j k l}=\left(\gamma_{4} \gamma_{I}\right)^{i(j}\left(\gamma_{4} \gamma_{I}\right)^{|k| l)}, E_{3 A}^{i j k l}=\left(\gamma_{I}\right)^{i(j}\left(\gamma_{I}\right)^{|k| l)}$,

where the subscripts 1 and 3 denote the $\mathbf{1}$ and $\mathbf{3}$, respectively, and $S$ and $A$ denote the exchange symmetry of the irrep. $I$ runs from 1 to $3 . E_{1}$ and $E_{3}$ consist of two terms, as required by hypercharge conservation. The UV particle for each irrep can be easily identified, e.g., as in Ref. [74].

Only three of the six PERs are linearly independent, as there are only three independent $H^{4} D^{4}$-type operators, conventionally taken to be $O_{S, n}, n=0,1,2$, defined in Ref. [75]. The convex hull of the PERs determines $\mathcal{C}$ as a 3D triangular cone, whose cross section is shown in Fig. 1. There are three ERs: $E_{1}, E_{1 S}$, and $E_{1 A}$. What can we learn from this cone? First, any UV-completable EFT must stay within this cone. Its three facets are, after matching to the Wilson coefficients, $C_{S, 0} \geq 0, C_{S, 0}+C_{S, 2} \geq 0$, and $C_{S, 0}+C_{S, 1}+C_{S, 2} \geq 0, C_{S, n}$ being the coefficients of $O_{S, n}$. These are precisely the positivity bounds obtained from elastic scatterings of superposed Higgs modes, albeit numerically [29]. Here we see that they are the strongest bounds, even going beyond elastic scatterings. (This, however, is not always true; see the $W$-boson case.) Second, the shape of the cone contains nontrivial information about the UV completion. Suppose the coefficients are experimentally measured and fall into the blue region. We can immediately deduce that a new particle (or a multiparticle state, for loop-level UV completions), which is a $\mathrm{SU}(2)_{L}$ singlet and has hypercharge 1, must exist and couple to $H H$, in order to generate $E_{1}$, because the convex hull of all other PERs does not contain this point. Similarly, if it falls in the red (green) or orange region, we know that a new particle that lives in the $1 S(1 A)$ representation must exist.

The W-boson polyhedral cone.-Our second example is the $W$ boson, which has two polarization modes and is 
charged under the 3 of $\mathrm{SU}(2)_{L}$. The projection operators for $\mathbf{3} \otimes \mathbf{3}=\mathbf{1} \oplus \mathbf{3} \oplus \mathbf{5}$ of $\mathrm{SU}(2)_{L}$ are

$$
\begin{aligned}
& P_{\alpha \beta \gamma \sigma}^{1}=\frac{1}{N} \delta_{\alpha \beta} \delta_{\gamma \sigma}, \quad P_{\alpha \beta \gamma \sigma}^{2}=\frac{1}{2}\left(\delta_{\alpha \gamma} \delta_{\beta \sigma}-\delta_{\alpha \sigma} \delta_{\beta \gamma}\right), \\
& P_{\alpha \beta \gamma \sigma}^{3}=\frac{1}{2}\left(\delta_{\alpha \gamma} \delta_{\beta \sigma}+\delta_{\alpha \sigma} \delta_{\beta \gamma}\right)-\frac{1}{N} \delta_{\alpha \beta} \delta_{\gamma \sigma},
\end{aligned}
$$

where $N=3$. For the $\mathrm{SO}(2)$ rotation around the forward direction, the projectors for $\mathbf{2} \otimes \mathbf{2}=\mathbf{1} \oplus \mathbf{1} \oplus \mathbf{2}$ are similar but with $N=2$. With these we can construct nine PERs, denoted as $E_{m, n}$, from the tensor product of the $m$ th $\mathrm{SO}(2)$ and the $n$th $\mathrm{SU}(2)_{L}$ projectors. Five of them are linearly independent. All except for $E_{3,3}$ are extremal. This immediately determines $\mathcal{C}$ as a $5 \mathrm{D}$ polyhedral cone with eight edges.

This example remarkably illustrates the efficiency of the extremal approach in constraining the physical EFT space. To compare with the positivity bound approach, we switch to the inequality representation and, after mapping to the operator coefficients, obtain

$$
\begin{aligned}
& C_{T, 2} \geq 0, \quad 4 C_{T, 1}+C_{T, 2} \geq 0, \\
& C_{T, 2}+8 C_{T, 10} \geq 0, \quad 8 C_{T, 0}+4 C_{T, 1}+3 C_{T, 2} \geq 0, \\
& 12 C_{T, 0}+4 C_{T, 1}+5 C_{T, 2}+4 C_{T, 10} \geq 0, \\
& 4 C_{T, 0}+4 C_{T, 1}+3 C_{T, 2}+12 C_{T, 10} \geq 0 .
\end{aligned}
$$

Again, the corresponding operators $O_{T, n}$ are defined in Refs. [75,76]. All these bounds except for $C_{T, 2} \geq 0$ have not appeared previously in the literature and are indeed stronger than those presented in Refs. [27,29]. These coefficients parameterize the anomalous quartic-gaugeboson couplings, currently being measured at the LHC [60-62], so they alone are important results. The first four bounds can be identified as positivity bounds by scattering various superposed states of $\left|W_{x, y}^{1,2}\right\rangle$ [superscripts for $\mathrm{SU}(2)_{L}$ and subscripts for polarization]. The last two bounds [Eqs. (10) and (11)] deserve more attention: They cannot be derived from any elastic scattering between superposed states, so they are beyond elastic positivity.

More than elastic positivity.-As explained already, elastic positivity fails to give a complete description of $\mathcal{C}$, because, in general, $\mathcal{C}^{*}$ contains more elements than $\mathcal{Q}$. The two bounds in Eqs. (10) and (11) are indeed from the following elements of $\mathcal{C}^{*}$, not contained in $\mathcal{Q}$ :

$$
\begin{array}{r}
T_{1}=6 E_{1,1}+3 E_{2,1}+6 E_{2,2}+3 / 2 E_{3,1}+3 E_{3,3}, \\
T_{2}=5 / 2 E_{1,1}+5 E_{1,2}+E_{1,3}+15 / 2 E_{2,1}+3 E_{3,3} .
\end{array}
$$

One can show that $T_{1,2}^{i j k l} M^{i j k l} \geq 0$, which lead to Eqs. (10) and (11), respectively, and that $T_{1,2} \notin \mathcal{Q}$, which implies that those bounds cannot be derived from scattering between superposed states (see Supplemental Material [66] for a proof with more details).

The fact that $T_{1,2} \notin \mathcal{Q}$ suggests that the dispersion relation of scattering amplitudes with entangled states can provide additional information about the UV completion. Positivity bounds would not capture this information unless there is a systematic and efficient way to tackle all elements in $\mathcal{C}^{*}$. Note that the $T_{1,2}$ tensors are independent of this specific problem and may lead to new bounds also for other operators or EFTs, whenever the number of states $n \geq 6$. Our extremal approach naturally captures all such cases.

The fermion circular cone.-Lastly, we consider SM-like chiral fermions, with left- and right-handed components carrying different hypercharges but other symmetries neglected for simplicity. Defining $J_{L, R}^{\mu} \equiv \bar{f}_{L, R} \gamma^{\mu} f_{L, R}$, we use the following basis:

$O_{1}=-\partial^{\mu} J_{L}^{\nu} \partial_{\mu} J_{L \nu}, \quad O_{2}=-\partial^{\mu} J_{R}^{\nu} \partial_{\mu} J_{R \nu}$,

$O_{3}=\partial^{\mu} J_{L}^{\nu} \partial_{\mu} J_{R \nu}, \quad O_{4}=D^{\mu}\left(\bar{f}_{L} f_{R}\right) D_{\mu}\left(\bar{f}_{R} f_{L}\right)$.

We simply show the PERs, in terms of the coefficient vector $\vec{C}=\left(C_{1}, C_{2}, C_{3}, C_{4}\right)$ :

$M_{L}:(1,0,0,0), \quad D_{S}:(0,0,0,1), \quad V:\left(1, r^{2},-2 r, 0\right)$,

$M_{R}:(0,1,0,0), \quad D_{A}:(0,0,-1,1), \quad V^{\prime}:(0,0,-1,2)$.

$M_{L, R}$ are from Majorana-type scalar couplings with two $f_{L}$ 's or two $f_{R}$ 's. $D$ is from a Dirac-type scalar coupling, with subscripts $S$ and $A$ indicating the exchange symmetry. $V\left(V^{\prime}\right)$ is from the vector coupling formed by same (opposite)-chirality fermions. $r$ is the ratio between $R / L$ couplings. Since $V$ is continuously parameterized by $r, \mathcal{C}$ has a curved boundary. In Fig. 2, we show a 3D slice of $\mathcal{C}$. The boundaries are given by $C_{1}, C_{2}, C_{4} \geq 0$ and $2 \sqrt{C_{1} C_{2}} \geq \max \left(C_{3},-C_{3}-C_{4}\right)$.

A geometric view for UV determination.-Let us reiterate what the Higgs example tells us in more general cases. Let $\mathcal{E}_{\backslash a}$ be the convex hull of all PERs with one of them, $\vec{E}_{a}$,

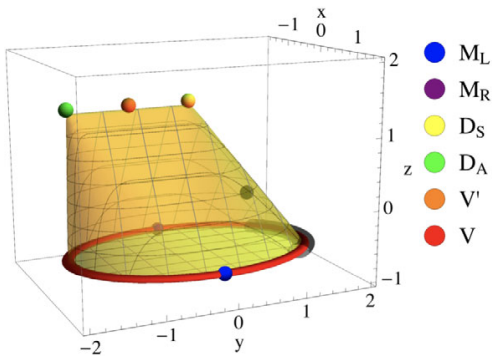

FIG. 2. A slice of the 4D fermion cone, taken to be perpendicular to the direction $(1,1,0,1)$. The three axes are taken to be $(1,-1,0,0),(0,0,1,0)$, and $(-1,-1,0,2)$. 
removed. If the measured coefficients, denoted as $\vec{C}_{\text {exp }}$, are not contained by $\mathcal{E}_{\backslash a}$, then a tree-level UV completion must contain a particle that couples with the $E_{a}$ irrep. This feature extends to loop-generated cases. For example, in the blue region in Fig. 1, there must exist some multiparticle state that couples to $H H$, carries hypercharge 1 , and contains a $\mathrm{SU}(2)_{L}$ singlet.

Quantitative statements can be made. For a measured $\vec{C}_{\text {exp }}$ in the blue region, there is a minimum $\lambda$ such that $\vec{C}_{\text {exp }}-\lambda \vec{E}_{1} \in \mathcal{E}_{\backslash 1}$. This sets a lower bound on the strength of the UV coupling that generates $\vec{E}_{1}$. Similarly, an upper bound can be set using $\vec{C}_{\text {exp }}-\lambda \vec{E}_{i} \in \mathcal{C}$ for all $\vec{E}_{i}$. As a second example, consider the fermion cone and assume $\vec{C}_{\text {exp }} \propto(1,0.8,1.4,1)$ is observed (see the black point in Fig. 2). If a small arc on $V$ (shown in black) is removed, the convex hull of remaining PERs does not contain $\vec{C}_{\text {exp }}$. It follows that a UV state exists and couples to the fermions with $V / A$-type couplings, and an upper bound on the coupling ratio $\left|g_{V} / g_{A}\right|<0.35$ can be set. There are many other interesting and phenomenologically relevant examples, where convex hulls can be used to infer UV states. This is not possible at dim-6, as the PERs would positively span the entire space.

As a final remark, we have shown that concepts and theorems in convex geometry help develop a deeper understanding of the EFT space, to improve the positivity bounds, and to determine the UV completion [77]. We hope that through this geometric perspective other results in convex geometry may find their applications in particle physics.

We thank Gauthier Durieux, Jiayin Gu, Yu-tin Huang, Fabio Maltoni, Jing Shu, Zi-Yue Wang, and Ming-Lei Xiao for helpful discussions and comments. C. Z. is supported by Institute for High Energy Physics, Chinese Academy of Sciences under Contract No. Y7515540U1 and by National Natural Science Foundation of China (NSFC) under Grant No. 12035008. S.-Y.Z. acknowledges support from the starting grants from University of Science and Technology of China under Grants No. KY2030000089 and No. GG2030040375 and is also supported by NSFC under Grants No. 11947301 and No. 12075233.

*cenzhang@ihep.ac.cn

† zhoushy@ustc.edu.cn

[1] S. Weinberg, Phenomenological Lagrangians, Physica (Amsterdam) 96A, 327 (1979).

[2] W. Buchmuller and D. Wyler, Effective Lagrangian analysis of new interactions and flavor conservation, Nucl. Phys. B268, 621 (1986).

[3] C. Ngoc Leung, S. T. Love, and S. Rao, Low-energy manifestations of a new interaction scale: Operator analysis, Z. Phys. C 31, 433 (1986).
[4] B. Henning, X. Lu, and H. Murayama, How to use the Standard Model effective field theory, J. High Energy Phys. 01 (2016) 023.

[5] A. Drozd, J. Ellis, J. Quevillon, and T. You, The universal one-loop effective action, J. High Energy Phys. 03 (2016) 180.

[6] B. Henning, X. Lu, and H. Murayama, One-loop matching and running with covariant derivative expansion, J. High Energy Phys. 01 (2018) 123.

[7] S. A. R. Ellis, J. Quevillon, T. You, and Z. Zhang, Mixed heavy-light matching in the universal one-loop effective action, Phys. Lett. B 762, 166 (2016).

[8] J. Fuentes-Martin, J. Portoles, and P. Ruiz-Femenia, Integrating out heavy particles with functional methods: A simplified framework, J. High Energy Phys. 09 (2016) 156.

[9] Z. Zhang, Covariant diagrams for one-loop matching, J. High Energy Phys. 05 (2017) 152.

[10] S. A. R. Ellis, J. Quevillon, T. You, and Z. Zhang, Extending the universal one-loop effective action: Heavy-light coefficients, J. High Energy Phys. 08 (2017) 054.

[11] M. Krmer, B. Summ, and A. Voigt, Completing the scalar and fermionic universal one-loop effective action, J. High Energy Phys. 01 (2020) 079.

[12] J. de Blas, J. C. Criado, M. Perez-Victoria, and J. Santiago, Effective description of general extensions of the Standard Model: The complete tree-level dictionary, J. High Energy Phys. 03 (2018) 109.

[13] J. C. Criado, MatchingTools: A Python library for symbolic effective field theory calculations, Comput. Phys. Commun. 227, 42 (2018).

[14] S. Das Bakshi, J. Chakrabortty, and S. Kumar Patra, CoDEx: Wilson coefficient calculator connecting SMEFT to UV theory, Eur. Phys. J. C 79, 21 (2019).

[15] By an EFT, we mean a set of operators with certain Wilson coefficients. The parameter space of (or simply space of) the EFT is spanned by possible values of all coefficients.

[16] A. Adams, N. Arkani-Hamed, S. Dubovsky, A. Nicolis, and R. Rattazzi, Causality, analyticity and an IR obstruction to UV completion, J. High Energy Phys. 10 (2006) 014.

[17] T. N. Pham and T. N. Truong, Evaluation of the derivative quartic terms of the meson chiral lagrangian from forward dispersion relation, Phys. Rev. D 31, 3027 (1985).

[18] M. R. Pennington and J. Portoles, The Chiral Lagrangian parameters, $l_{1}, l_{2}$, are determined by the $\rho$ resonance, Phys. Lett. B 344, 399 (1995).

[19] B. Ananthanarayan, D. Toublan, and G. Wanders, Consistency of the chiral pion pion scattering amplitudes with axiomatic constraints, Phys. Rev. D 51, 1093 (1995).

[20] J. Comellas, J. I. Latorre, and J. Taron, Constraints on chiral perturbation theory parameters from QCD inequalities, Phys. Lett. B 360, 109 (1995).

[21] A. V. Manohar and V. Mateu, Dispersion relation bounds for $\pi \pi$ scattering, Phys. Rev. D 77, 094019 (2008).

[22] B. Bellazzini, Softness and amplitudes' positivity for spinning particles, J. High Energy Phys. 02 (2017) 034.

[23] C. de Rham, S. Melville, A. J. Tolley, and S.-Y. Zhou, Positivity bounds for scalar field theories, Phys. Rev. D 96, 081702(R) (2017). 
[24] C. de Rham, S. Melville, A. J. Tolley, and S.-Y. Zhou, UV complete me: Positivity bounds for particles with spin, J. High Energy Phys. 03 (2018) 011.

[25] N. Arkani-Hamed, Y. Huang, and T.-C. Huang (to be published).

[26] C. Zhang and S.-Y. Zhou, Positivity bounds on vector boson scattering at the LHC, Phys. Rev. D 100, 095003 (2019).

[27] Q. Bi, C. Zhang, and S.-Y. Zhou, Positivity constraints on aQGC: Carving out the physical parameter space, J. High Energy Phys. 06 (2019) 137.

[28] B. Bellazzini and F. Riva, New phenomenological and theoretical perspective on anomalous $\mathrm{ZZ}$ and $\mathrm{Z} \gamma$ processes, Phys. Rev. D 98, 095021 (2018).

[29] G. N. Remmen and N. L. Rodd, Consistency of the standard model effective field theory, J. High Energy Phys. 12 (2019) 032.

[30] G. N. Remmen and N. L. Rodd, Flavor Constraints from Unitarity and Analyticity, Phys. Rev. Lett. 125, 081601 (2020).

[31] C. de Rham, S. Melville, A. J. Tolley, and S.-Y. Zhou, Positivity bounds for massive spin-1 and spin-2 fields, J. High Energy Phys. 03 (2019) 182.

[32] C. de Rham, S. Melville, A. J. Tolley, and S.-Y. Zhou, Massive Galileon positivity bounds, J. High Energy Phys. 09 (2017) 072.

[33] D. Baumann, D. Green, H. Lee, and R. A. Porto, Signs of analyticity in single-field inflation, Phys. Rev. D 93, 023523 (2016).

[34] B. Bellazzini, C. Cheung, and G. N. Remmen, Quantum gravity constraints from unitarity and analyticity, Phys. Rev. D 93, 064076 (2016).

[35] C. Cheung and G. N. Remmen, Positive signs in massive gravity, J. High Energy Phys. 04 (2016) 002.

[36] C. Cheung and G. N. Remmen, Positivity of CurvatureSquared Corrections in Gravity, Phys. Rev. Lett. 118, 051601 (2017).

[37] B. Bellazzini, F. Riva, J. Serra, and F. Sgarlata, Beyond Positivity Bounds and the Fate of Massive Gravity, Phys. Rev. Lett. 120, 161101 (2018).

[38] J. Bonifacio, K. Hinterbichler, and R. A. Rosen, Positivity constraints for pseudolinear massive spin-2 and vector Galileons, Phys. Rev. D 94, 104001 (2016).

[39] K. Hinterbichler, A. Joyce, and R. A. Rosen, Massive spin-2 scattering and asymptotic superluminality, J. High Energy Phys. 03 (2018) 051.

[40] J. Bonifacio, K. Hinterbichler, A. Joyce, and R. A. Rosen, Massive and massless spin-2 scattering and asymptotic superluminality, J. High Energy Phys. 06 (2018) 075.

[41] B. Bellazzini, F. Riva, J. Serra, and F. Sgarlata, The other effective fermion compositeness, J. High Energy Phys. 11 (2017) 020.

[42] J. Bonifacio and K. Hinterbichler, Bounds on amplitudes in effective theories with massive spinning particles, Phys. Rev. D 98, 045003 (2018).

[43] B. Bellazzini, M. Lewandowski, and J. Serra, Positivity of Amplitudes, Weak Gravity Conjecture, and Modified Gravity, Phys. Rev. Lett. 123, 251103 (2019).

[44] S. Melville and J. Noller, Positivity in the Sky: Constraining dark energy and modified gravity from the UV, Phys. Rev. D 101, 021502(R) (2020).
[45] C. de Rham and A.J. Tolley, The speed of gravity, Phys. Rev. D 101, 063518 (2020).

[46] L. Alberte, C. de Rham, A. Momeni, J. Rumbutis, and A. J. Tolley, Positivity constraints on interacting spin-2 fields, J. High Energy Phys. 03 (2020) 097.

[47] L. Alberte, C. de Rham, A. Momeni, J. Rumbutis, and A. J. Tolley, Positivity constraints on interacting pseudolinear spin-2 fields, J. High Energy Phys. 07 (2020) 121.

[48] G. Ye and Y.-S. Piao, Positivity in the effective field theory of cosmological perturbations, Eur. Phys. J. C 80, 421 (2020).

[49] M. Herrero-Valea, I. Timiryasov, and A. Tokareva, To positivity and beyond, where Higgs-Dilaton inflation has never gone before, J. Cosmol. Astropart. Phys. 11 (2019) 042.

[50] Y.-J. Wang, F.-K. Guo, C. Zhang, and S.-Y. Zhou, Generalized positivity bounds on chiral perturbation theory, J. High Energy Phys. 07 (2020) 214.

[51] B. Henning, X. Lu, T. Melia, and H. Murayama, 2, 84, 30, 993, 560, 15456, 11962, 261485, ...: Higher dimension operators in the SM EFT, J. High Energy Phys. 08 (2017) 016; Erratum, J. High Energy Phys. 09 (2019) 019(E).

[52] C. W. Murphy, Dimension-8 operators in the standard model effective field theory, arXiv:2005.00059.

[53] H.-L. Li, Z. Ren, J. Shu, M.-L. Xiao, J.-H. Yu, and Y.-H. Zheng, Complete set of dimension-8 operators in the standard model effective field theory, arXiv:2005 .00008 .

[54] D. Liu, A. Pomarol, R. Rattazzi, and F. Riva, Patterns of strong coupling for LHC searches, J. High Energy Phys. 11 (2016) 141.

[55] A. Azatov, R. Contino, C. S. Machado, and F. Riva, Helicity selection rules and noninterference for BSM amplitudes, Phys. Rev. D 95, 065014 (2017).

[56] J. Ellis and S.-F. Ge, Constraining Gluonic Quartic Gauge Coupling Operators with $g g \rightarrow \gamma \gamma$, Phys. Rev. Lett. 121, 041801 (2018).

[57] C. Hays, A. Martin, V. Sanz, and J. Setford, On the impact of dimension-eight SMEFT operators on Higgs measurements, J. High Energy Phys. 02 (2019) 123.

[58] J. Ellis, S.-F. Ge, H.-J. He, and R.-Q. Xiao, Probing the scale of new physics in the $Z Z \gamma$ coupling at $e^{+} e^{-}$colliders, Chin. Phys. C 44, 063106 (2020).

[59] S. Alioli, R. Boughezal, E. Mereghetti, and F. Petriello, Novel angular dependence in Drell-Yan lepton production via dimension-8 operators, Phys. Lett. B 809, 135703 (2020).

[60] A. M. Sirunyan et al., Search for anomalous electroweak production of vector boson pairs in association with two jets in proton-proton collisions at $13 \mathrm{TeV}$, Phys. Lett. B 798, 134985 (2019).

[61] CMS Collaboration, Measurements of production cross sections of same-sign WW and WZ boson pairs in association with two jets in proton-proton collisions at $\sqrt{s}=13 \mathrm{TeV}$, Phys. Lett. B 809, 135710 (2020).

[62] A. M. Sirunyan et al. (CMS Collaboration), Measurement of the cross section for electroweak production of a $\mathrm{Z}$ boson, a photon and two jets in proton-proton collisions at $\sqrt{\mathrm{s}}=$ $13 \mathrm{TeV}$ and constraints on anomalous quartic couplings, J. High Energy Phys. 06 (2020) 076. 
[63] P. Azzi et al., Standard Model Physics at the HL-LHC and HE-LHC, arXiv:1902.04070.

[64] R. S. Gupta, Liberating Higgs/EW observables at dimension 8, Proceedings of the Workshop Higgs and Effective Field Theory 2020 (2020) https://indico.cern.ch/ event/855352/contributions/3759834/attachments/2020293/ 3377866/heft2020.pdf.

[65] M. Krein and D. Milman, On extreme points of regular convex sets, Stud. Math. 9, 133 (1940).

[66] See Supplemental Material at http://link.aps.org/ supplemental/10.1103/PhysRevLett.125.201601 for more details about how to derive the dispersion relation, how to get the bounds in Eqs. (10) and (11) from $T_{1,2}$ given in Eqs. (12) and (13), and why these bounds can not be derived from the positivity bounds of scattering between superposed states.

[67] S. Andriolo, T.-C. Huang, T. Noumi, H. Ooguri, and G. Shiu, Duality and axionic weak gravity, Phys. Rev. D 102, 046008 (2020).

[68] K. Yamashita, C. Zhang, and S.-Y. Zhou, Elastic positivity vs extremal positivity bounds in SMEFT: A case study in transversal electroweak gauge-boson scatterings, arXiv: 2009.04490.

[69] H. Minkowski, Geometry of Numbers. (Geometrie der Zahlen.), Bibliotheca Mathematica Teubneriana. 40 (Johnson Reprint Corp. vii, New York, NY, 1968), p. 256.

[70] H. Weyl, Elementare theorie der konvexen polyeder, Commentarii mathematici Helvetici 7, 290 (1935).
[71] D. Avis and K. Fukuda, A pivoting algorithm for convex hulls and vertex enumeration of arrangements and polyhedra, Discrete Comput. Geom. 8, 295 (1992).

[72] D. Avis, http://cgm.cs.mcgill.ca/ avis/C/lrs.html.

[73] A. Helset, M. Paraskevas, and M. Trott, Gauge fixing the Standard Model Effective Field Theory, Phys. Rev. Lett. 120, 251801 (2018).

[74] I. Low, R. Rattazzi, and A. Vichi, Theoretical constraints on the Higgs effective couplings, J. High Energy Phys. 04 (2010) 126.

[75] C. Degrande, O. Eboli, B. Feigl, B. Jger, W. Kilian, O. Mattelaer, M. Rauch, J. Reuter, M. Sekulla, and D. Wackeroth, Monte Carlo tools for studies of non-standard electroweak gauge boson interactions in multi-boson processes: A Snowmass White Paper, in Proceedings of the 2013 Community Summer Study on the Future of U.S. Particle Physics: Snowmass on the Mississippi (CSS2013): Minneapolis, MN, USA, 2013 (2013), https://www.slac .stanford.edu/econf/C1307292/docs/SnowmassBook.pdf.

[76] $C_{T, 10}$ denotes the coefficient of $O_{2}^{W^{4}}$ of Ref. [29], multiplied by $g_{2}^{4} / 4$.

[77] The EFThedron of Ref. [25] also connects the UV states in a geometric point of view. Convex objects such as cyclic polytopes are found to constrain sequences of operators with increasing dimensions. Here we consider EFTs endowed with symmetries and focus on operators with lowest dimensions. 